\title{
Experimental Evidence of an Optical Shutter in Cholesteric Phase of a Double Hydrogen Bonded Liquid Crystal
}

\author{
V.N. Vijayakumar and M.L.N. Madhu Mohan* \\ Liquid Crystal Research Laboratory (LCRL), Bannari Amman Institute of Technology, Sathyamangalam 638 401, India
}

(Received on 14 August, 2009)

\begin{abstract}
A novel series of double hydrogen bonded liquid crystals have been isolated. Hydrogen bond was formed between non mesogen chiral ingredient levo tartaric acid (LTA) and mesogenic undecyloxy benzoic acids (11BA) Thermal and electrical properties exhibited by levo tartaric acid and undecyloxy benzoic acid (LTA+11BA) were discussed. Interesting feature of the present investigation was observation of an optical shuttering action in LTA+11BA hydrogen bonded complex on application of a stipulated applied dc bias voltage. By enhancement of the dc bias voltage the mesogen behaves like an optical shutter, thus this hydrogen bonded complex mesogen acts as an effective light modulator. It was noticed that this action of shuttering was reversible, in the sense that when applied bias voltage was removed the original texture was restored. Experimental results relating to textures, optical tilt, dielectric studies and optical shuttering action were presented. This optical shutter property of the mesogen can be exploited for commercial and display device applications.
\end{abstract}

Keywords: Hydrogen bonded liquid crystal, LTA+11BA, Optical shutter, Light modulator

\section{Introduction}

'Liquid Crystal' (LC) materials refer to the fourth state of matter that posses dual property of crystals and liquids. Their anisotropic physical properties (characteristic of its crystalline nature) and the inherent surface alignment (to the substrate) tendency (characteristic of its fluidity) are extensively exploited [1-3] to design LC based Electro-Optic devices. Their overall shape [4] being cylindrical, renders them readily viable for surface alignment. In the field of supramolecular LCs, especially in the field of hydrogen bonded LCs, the influence of soft-covalent interaction for the thermal stability of phases of device interest is widely [2-14] studied.

The configuration and spread of the hydrogen bonding interaction with regard to the long molecular dipole moment in the calamitic LCs along with the corresponding molecular dipole models are argued $[5,7,8]$ to originate the distinct properties in the family of hydrogen bonded liquid crystals (HBLC). However, as an integrated overview, for the microscopic interactions among molecular dipoles as contributed by the underlying chemical moieties configured on the molecular body (or frame) would be of immense utility in devices. The field of 'Systematic Studies' in the area of LCs is expected to further reveal the truth of microscopic interactions. The growth of order (and crystalline nature) is logically expected to increase with decreasing temperature in these LC materials in their condensed version of matter.

In the case of HBLCs the molecular frame possesses [10, 18-22] additional soft-covalent interaction. Further, in case of non-ferroelectric and ferroelectric HBLCs, the observed $[13,21]$ variation in device relevant properties like thermal stability, spontaneous polarization, tilt angle, switching times etc., are addressed through dipolar and refined dipolar models to vouch the fact that soft-covalent interaction extends rather inclined to the long molecular dipole moment. Further, the presence of additional interaction in the form of anisotropic dipole moment along the molecular body is believed to give origin to the orientational disorder leading to tilted phases.

${ }^{*}$ Electronic address: mln.madhuegmail.com
With our previous experience [23-30], in designing, synthesizing and characterizing various types of liquid crystals, a successful attempt has been made in characterizing a novel series of inter hydrogen bonded liquid crystal exhibiting optical shutter activity in cholesteric phase of LTA+11BA hydrogen bonded complex.

In the present communication a homologous series of HBLC is designed in such a way that the molecule possesses double hydrogen bonding with chiral ingredient levo tartaric acid moiety. Thermal and electrical characterizations of undecyloxy benzoic acid and levo tartaric acid complex $(\mathrm{LTA}+11 \mathrm{BA})$ pertaining to the present series are discussed in detail.

\section{Experimental}

Optical textural observations are made with a Nikon polarizing microscope equipped with Nikon digital CCD camera system with 5 mega pixels and $2560 * 1920$ pixel resolutions. The liquid crystalline textures are processed, analyzed and stored with the aid of ACT-2U imaging software system. The temperature control of the liquid crystal cell is equipped by Instec HCS402-STC 200 temperature controller (Instec, USA) to a temperature resolution of $\pm 0.1^{\circ} \mathrm{C}$. This unit is interfaced to computer by IEEE -STC 200 to control and monitored the temperature. The liquid crystal sample is filled by capillary action in its isotropic state into a commercially available (Instec, USA) polyamide buffed cell with 4 micron spacer. Optical extinction technique is used for the determination of tilt angle. The transition temperatures and corresponding enthalpy values are obtained by DSC (Shimadzu DSC-60). FTIR spectra has been recorded (ABB FTIR MB3000) and analyzed with the MB3000 software. Light intensity measurements where made with photo transistor (TSL 252) where the sample is placed under crossed polarizers of the Nikon polarizing microscope. The p-n-alkoxy benzoic acids (nABA) and levo tartaric acid were supplied by Sigma Aldrich, Germany and all the solvents used are E.Merk grade. 


\section{Synthesis of HBLC}

The present intermolecular hydrogen bonded mesogen is synthesized by the addition of two moles of undecyloxy benzoic acids (11BA) with one mole of levo tartaric acid (LTA) in N,N-Dimethyl formamide (DMF) respectively. Further it has been subjected to constant stirring for 10 hours at ambient temperature of $30^{\circ} \mathrm{C}$ till a white precipitate in a dense solution is formed. The white crystalline crude complexes so obtained by removing excess DMF are then recrystallized with Dimethyl Sulfoxide (DMSO) and the yield varied from $85 \%$ to $95 \%$. The general molecular structure of the present homologous series of p-n-alkoxy benzoic acids with levo tartaric acid is depicted in the figure 1 , where $\mathrm{n}$ represents the alkoxy carbon number.

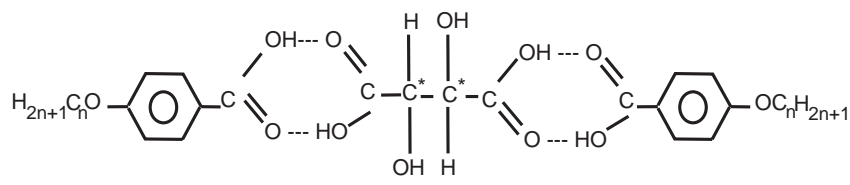

FIG. 1: General molecular diagram of LTA+nBA homologous series

\section{Results and Discussion}

Levo tartaric acid and undecyloxy benzoic acid complex (LTA+11BA) isolated are white crystalline solids and are stable at room temperature. It is insoluble in water and sparingly soluble in common organic solvents such as methanol, ethanol, and benzene and dicholoro methane. However it shows a high degree of solubility in coordinating solvents like dimethyl sulfoxide (DMSO) and pyridine. It melts at specific temperatures below $140^{\circ} \mathrm{C}$. Further it shows high thermal and chemical stability when subjected to repeated thermal scans performed during polarizing microscopic studies (POM) and differential scanning calorimetric (DSC) studies.

\section{Infrared Spectroscopy (FTIR)}

IR spectra of free p-n-alkoxy benzoic acid, levo tartaric acid and their intermolecular hydrogen bonded complexes are recorded in the solid state $(\mathrm{KBr})$ at room temperature. Figure 2 illustrates the FTIR spectra of the hydrogen bonded complex of LTA+11BA in solid state at room temperature. The solid state spectra of free alkoxy benzoic acid is reported [21] to have two sharp bands at $1685 \mathrm{~cm}^{-1}$ and $1695 \mathrm{~cm}^{-1}$ due to the frequency $\mathrm{v}(\mathrm{C}=\mathrm{O})$ mode. The doubling feature of this stretching mode confirms the dimeric nature of alkoxy benzoic acid at room temperature [21]. Further in the present LTA+11BA hydrogen bonded complex a band appearing at $2924 \mathrm{~cm}^{-1}$ is assigned to $\mathrm{v}(\mathrm{O}-\mathrm{H})$ mode of the carboxylic acid group.

The doubling nature of $\mathrm{v}(\mathrm{C}=\mathrm{O})$ mode may be attributed to the dimeric nature of acid group at room temperature [21] Corresponding spectrum of solution state (chloroform) show a strong intense band suggesting the existence of monomeric

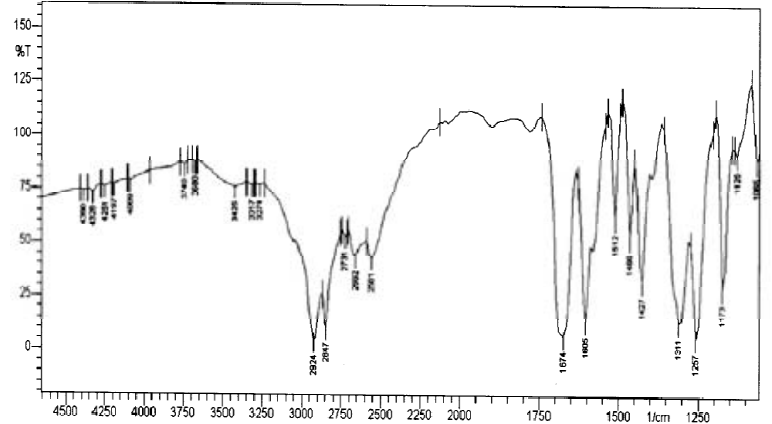

FIG. 2: FTIR spectrum of LTA+11BA hydrogen bonded complex

form of benzoic acid. A noteworthy feature in the spectra of LTA+11BA complex is the appearance of a broad band at $1674 \mathrm{~cm}^{-1}$ and non appearance of the doubling nature of $v(\mathrm{C}=\mathrm{O})$ mode of benzoic acid moiety. This clearly suggests that the dimeric nature of the benzoic acid dissociates and prefers to exist in a monomeric form upon complexation.

\section{Phase Identification}

The observed phase variants, transition temperatures and corresponding enthalpy values obtained by DSC in cooling and heating cycles for the LTA+11BA complex are presented in table 1 .

\begin{tabular}{|c|c|c|c|c|c|c|c|c|}
\hline Complex & $\begin{array}{c}\text { Phase } \\
\text { variance }\end{array}$ & \multicolumn{2}{|c|}{ Technique } & $\begin{array}{c}\text { Crystal } \\
\text { Melt }\end{array}$ & Ch & $\mathrm{C}^{*}$ & $\mathrm{G}^{*}$ & Crystal \\
\hline \multirow{5}{*}{$\mathrm{LTA}+11 \mathrm{BA}$} & \multirow{5}{*}{$\mathrm{ChC}^{*} \mathrm{G}^{*}$} & \multicolumn{2}{|c|}{ DSC (h) } & $\begin{array}{l}97.84 \\
(47.68)\end{array}$ & $\begin{array}{l}140.21 \\
(2.33)\end{array}$ & $\begin{array}{l}129.37 \\
(2.47)\end{array}$ & \# & \\
\hline & & \multicolumn{2}{|c|}{$\operatorname{DSC}(\mathrm{c})$} & & $\begin{array}{l}136.78 \\
(2.96)\end{array}$ & $\begin{array}{l}125.5 \\
(2.12)\end{array}$ & $\begin{array}{l}84.84 \\
(8.55)\end{array}$ & $\begin{array}{l}72.54 \\
(12.81)\end{array}$ \\
\hline & & \multicolumn{2}{|c|}{ POM (c) } & & 137.1 & 126.2 & 85.3 & 72.9 \\
\hline & & \multirow{2}{*}{$\begin{array}{l}\text { Dielectric } \\
\text { Studies (c) }\end{array}$} & $10 \mathrm{KHz}$ & & 134.9 & 128.2 & 87.3 & 69.0 \\
\hline & & & $100 \mathrm{KHz}$ & & 135 & 128.4 & 87.3 & 68.9 \\
\hline
\end{tabular}

TABLE 1: Comparison of transition temperatures obtained by different techniques

\section{LTA+nBA Homologous Series}

The levo tartaric acid and alkoxy benzoic acid homologous complex is found to exhibit characteristic textures [31], viz., Cholesteric (finger print texture), Smectic $\mathrm{C}^{*}$ (schlieren texture) and Smectic $G^{*}$ (multi colored mosaic texture) respectively. The phase sequence of the levo tartaric acid and undecyloxy benzoic acid (LTA+11BA) hydrogen bonded complex in the cooling run can be shown as:

$$
\text { Isotropic } \rightarrow \mathrm{Ch} \rightarrow \mathrm{Sm}^{*} \rightarrow \mathrm{SmG}^{*} \rightarrow \text { Crystal }
$$




\section{DSC Studies}

DSC thermo grams are recorded in heating and cooling cycles. The liquid crystal sample is crimped in an aluminum cell and heated with a scan rate of $10^{\circ} \mathrm{C} / \mathrm{min}$ and hold at its isotropic temperature for one minute so as to attain thermal stability. The cooling run is performed with an identical scan rate of $10^{\circ} \mathrm{C} / \mathrm{min}$. DSC thermo gram of LTA+11BA is illustrated as figure 3 . The respective equilibrium transition temperatures and corresponding enthalpy values of the mesogens are listed separately in table 1. POM studies are good agreement with the DSC results.

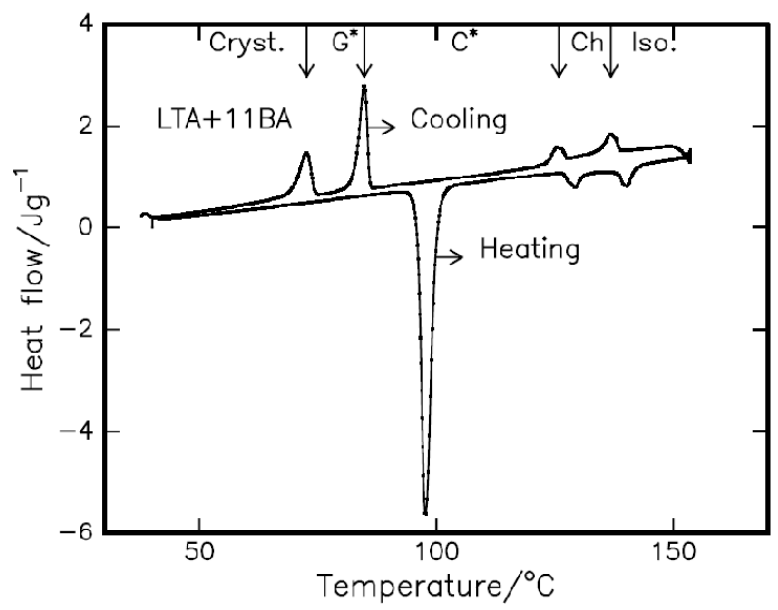

FIG. 3: DSC thermo gram of LTA+11BA complex recorded with a scan rate of $100 \mathrm{C} / \mathrm{min}$

\section{Phase diagrams}

The phase diagrams of pure p-n-alkoxy benzoic acids and the present homologous series (nBA+LTA) are constructed and reported [30] through optical polarizing microscopic studies by the phase transition temperatures observed in the cooling run of the mesogens of the present homologous series. The phase diagram of pure p-n-alkoxy benzoic acids is reported [24] to exhibit three phases namely, Nematic, smectic C* and smectic $\mathrm{G}^{*}$.

\section{Optical Tilt Angle Studies}

For the hydrogen bonded LTA+11BA complex, optical tilt angle has been experimentally measured by optical extinction method [32] in the smectic $C^{*}$ phases. From the figure 4 it is observed that the tilt angle increases with decreasing temperature and attains a saturation value of $29^{\circ}$. The large magnitudes of the tilt angle are attributed [22] to the direction of the soft covalent hydrogen bond interaction which spreads along molecular long axis with finite inclination. Tilt angle is a primary order parameter [32,33] in tilted phases namely smectic $C^{*}$ The temperature variation is estimated by fitting

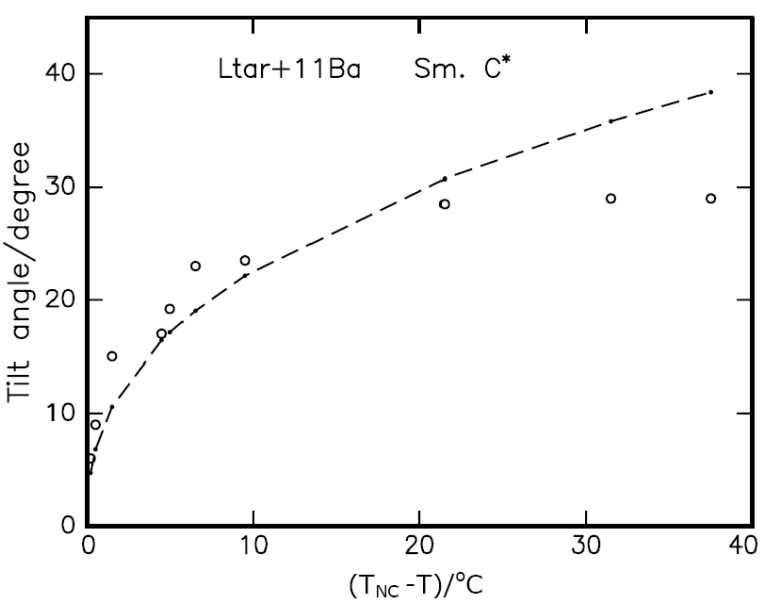

FIG. 4: Temperature dependence of optical tilt in smectic $C^{*}$ phase. Solid line denotes the fit.

the observed data of $\theta(\mathrm{T})$ to the relation

$$
\theta(T) \alpha\left(T-T_{C}\right)^{\beta}
$$

The critical exponent $\beta$ value estimated by fitting the data of $\theta(\mathrm{T})$ to the above equation (1) is found to be 0.50 to agree with the Mean Field [34] prediction. The dotted line in the figure 4 depicts the fitted data. Further, the agreement of $\beta$ with Mean Field value infers the long-range interaction of transverse dipole moment for the stabilization of tilted smectic C* phase.

\section{Dielectric Studies}

Dielectric studies enable to detect a second order transition which goes unobserved in the DSC study. Variation of capacitance and dielectric loss with temperature is performed for LTA $+11 \mathrm{BA}$ of the present homologous series. The mesogen filled in a commercially available 4 micron polyamide buffed cell (Instec) and is excited with a frequency of $10 \mathrm{KHz}$ and $100 \mathrm{KHz}$ respectively. Silver leads are drawn from the cell for electrical contacts. Simultaneous textural observations are made to co-relate the phase exhibited by the mesogen to the dielectric data. Thus the textural transition temperatures and dielectric spectrum are studied together. The dielectric spectrum of undecyloxy benzoic acid complex (LTA+11BA) as depicted in figure 5 is discussed below.

From figure 5 the following points can be inferred.

(a) As the temperature is decreased from isotropic, a small knick is observed at $134.9^{\circ} \mathrm{C}$ indicating the onset of the cholesteric phase. Cholesteric finger print texture is observed indicating the onset of the phase.

(b) On further decrement of the temperature the stabilization of the Cholesteric phase is evinced where the capacitance started increasing with out any sudden anomaly in the dielectric spectrum. 


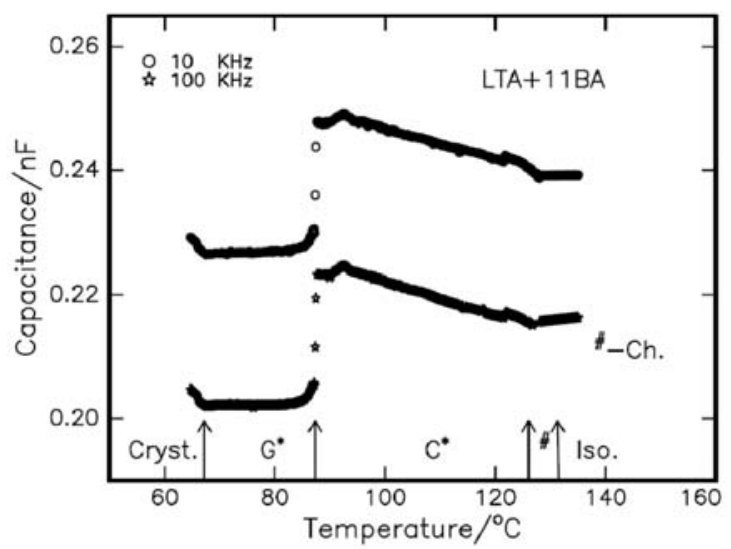

FIG. 5: Temperature dependence of capacitance at $10 \mathrm{KHz}$ and 100 $\mathrm{KHz}$

(c) At $128.2^{\circ} \mathrm{C}$ a small anomaly in the magnitude of the capacitance indicates the onset of smectic $\mathrm{C}^{*}$ phase. Schlieren texture is observed with the onset of this phase.

(d) In the entire region of smectic $\mathrm{C}^{*}$ phase a linear increment in the magnitude of the capacitance is observed indicating the growth and stabilization of the phase.

(e) Smectic $G^{*}$ phase is observed as a sudden steep fall in the magnitude of capacitance at $87.3^{\circ} \mathrm{C}$ indicates the transition from smectic $\mathrm{C}^{*}$ to smectic $\mathrm{G}^{*}$ phase. A multi colored mosaic texture is observed indicating the onset of smectic $\mathrm{G}^{*}$ phase

(f) Further decrement in temperature showed an unaltered variation of the capacitance indicating the stabilization of smectic $\mathrm{G}^{*}$ phase.

(g) A sudden variation in the magnitude of the capacitance at $69.9^{\circ} \mathrm{C}$ is attributed to the transition from the mesogenic phase to crystal. The formation of crystal is also evinced through optical textural studies.

Similar variations are observed in dielectric loss spectrum. The transition temperatures obtained by this technique are in good agreement with POM and DSC studies and are tabulated in table 1 .

\section{Dielectric relaxations}

Dielectric dispersion i.e. frequency variation of dielectric loss exhibited by LTA $+11 \mathrm{BA}$ is studied at different temperatures in smectic $\mathrm{C}^{*}$ phases in the frequency range of $5 \mathrm{~Hz}$ to $13 \mathrm{MHz}$ respectively. An impedance analyzer (HP4192A) is operated with $1 \mathrm{~V}_{P-P}$ oscillating signal with zero bias field. Relative permittivity $\varepsilon^{\prime} \mathrm{r}(\omega)$ and dielectric loss $\varepsilon$ " $(\omega)$ are calculated by the following equations

$$
\begin{aligned}
& * \varepsilon \operatorname{r}(\omega)=\varepsilon^{\prime} \mathrm{r}(\omega)-\mathrm{j} \varepsilon "(\omega) \\
& \varepsilon^{\prime} \mathrm{r}(\omega)=\left[\mathrm{C}_{L C}-\mathrm{C}_{\text {leads }}\right] /\left[\mathrm{C}_{\text {empty }}-\mathrm{C}_{\text {leads }}\right]
\end{aligned}
$$

$$
* \varepsilon \operatorname{r}(\omega)=\operatorname{Tan} \delta(\omega) * \varepsilon r(\omega)
$$

To detect the possible relaxation in the HBFLC complex, the mesogen is scanned in the frequency range of $5 \mathrm{~Hz}$ to $13 \mathrm{MHz}$ at different temperatures corresponding to smectic $\mathrm{C}^{*}$ phases. LTA+11BA mesogen exhibits a relaxation mechanism namely Goldstone Mode (GM).

\section{Goldstone mode}

Goldstone mode in smectic C* phase of LTA+11BA complex is studied and analyzed. As reported [22] in HBFLC, Goldstone mode is observed at lower frequencies namely at around $950 \mathrm{~Hz}$ in LTA+11BA complex. The variation of the capacitance with corresponding dielectric loss at various temperature which are referred as Cole-Cole plots, in smectic $\mathrm{C}^{*}$ for the LTA+11BA are shown in figure 6 and is interpreted as due to collective response originated due to the excitation of the coupled transverse dipole moment situated around the chiral centers over the layers in the form of polarization helix. This relaxation is identified [35] as Goldstone mode. The order of $f_{r}$ relevant to the GM in the present compounds are found to be comparable to that reported $[36,22]$ in other FLCs and HBFLC compounds. It is further observed that GM relaxation is suppressed under different applied fields as depicted in figure 7, thus this experimental evidence conforms that GM is originated from the helix spread over the smectic layers of respective phase.

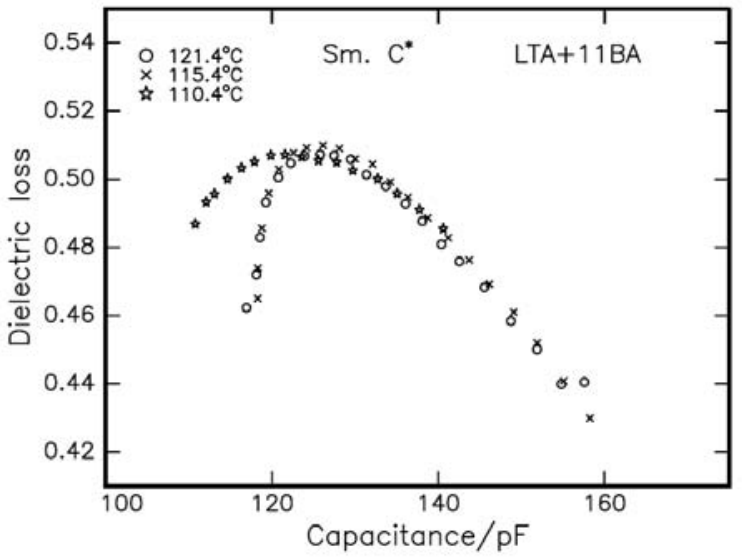

FIG. 6: Cole-Cole plots in smectic $C^{*}$ phase at various temperatures

\section{Field Induced transition (FiT)}

It is reported [29, 35-43] that when a mesogen is subjected to an applied external field in cholesteric, nematic or smectic $C^{*}$ phase there can be a phase transition which is referred as field induced transition (FiT). The present hydrogen bonded complex is subjected to various strengths of external dc bias voltage derived from HP 4192A impedance analyzer, to investigate the occurrence of FiT. 


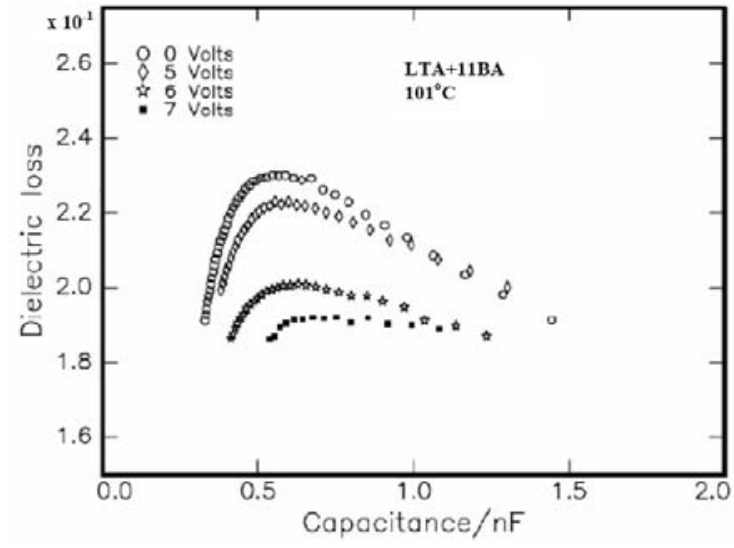

FIG. 7: Suppression of the helix under various applied external bias in the Goldstone mode of smectic $\mathrm{C}^{*}$ phase

\section{Optical shutter action in LTA+11BA}

LTA+11BA compound in its cholesteric phase when an applied dc bias voltage exceeds a particular threshold value, the phase of the compound is observed to prefer homeotropic like alignment with light being optically extinct which is referred as optical shutter. Plates 1 to 4 depict the cholesteric texture under the influence of various applied fields, further, it is interesting to note that immediately after withdrawing the bias voltage from any of the induced transition the original texture of the cholesteric phase is retained. Thus this process is reversible with bias voltage. In the entire thermal span of cholesteric phase ( $\sim 136^{\circ} \mathrm{C}$ to $\left.\sim 125^{\circ} \mathrm{C}\right)$ this is observed. While in the other phases preceding and succeeding cholesteric phase no such transition is found. Thus the filed induced transitions can be represented as

$$
E_{0} \rightarrow \text { Optical shutter }\left(E_{1}\right)
$$

A quantitative approach has been made to study the effect of applied electric field on hydrogen bonded LTA+11BA with optical textural studies and dielectric studies. The cholesteric phase with a bias voltage (both polarities) less than or equal to \pm 3.75 volts/micron is referred as $\mathrm{E}_{0}$ where there is no change in the texture of the phase as depicted in plate 1. As the voltage is increased in steps of 1.25 volts/micron the intensity of the light from the texture drops as can be seen from plates 2 and 3 .

An important observation is that when a dc bias voltage of \pm 5.0 volts/micron is applied, the optical extinction is observed with the optical texture of the compound suddenly disappears, this new phase is designated as optical shutter $\left(E_{1}\right)$ which is depicted as plate 4 . One of the possible reasons for this interesting phenomenon may be the realignment of the molecules to form a homeotropic like alignment.

To substantiate the above results dielectric studies are performed. The variation of capacitance with respect to various external bias voltages is studied in cholesteric phase. Such a variation for LTA+11BA compound is shown in figure 8 . The plot depicts a symmetric variation of the capacitance with respect to both positive and negative applied bias voltages. From the above figure 8 , it can be inferred that

a) Capacitance variation is identical for both the polarities inferring no influence of polarity.

b) Field induced transition i.e. $E_{0}-E_{1}$ (optical shutter), are not sudden and abrupt but smooth and uniform.

c) Frequency has no influence on the threshold values of the FiT.

d) Equilibrium states for the low and the high field are found to be distinct.

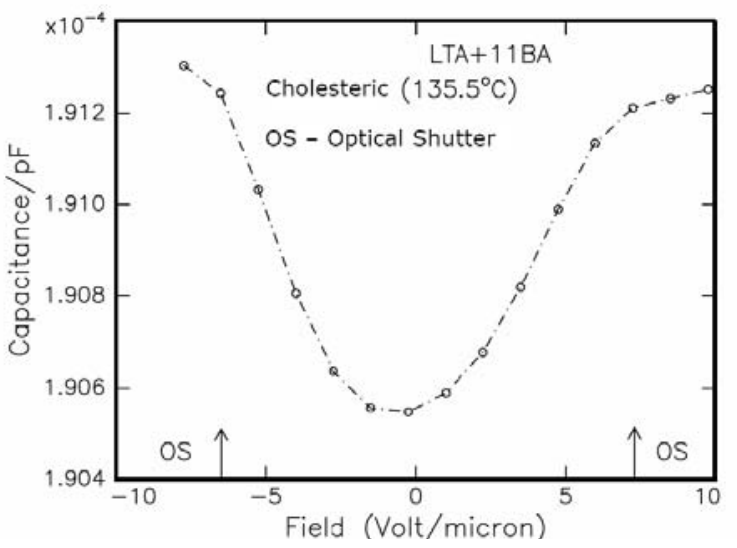

FIG. 8: Field dependence of capacitance in the cholesteric phase of LTA+11BA. Optical Shutter is denoted as OS

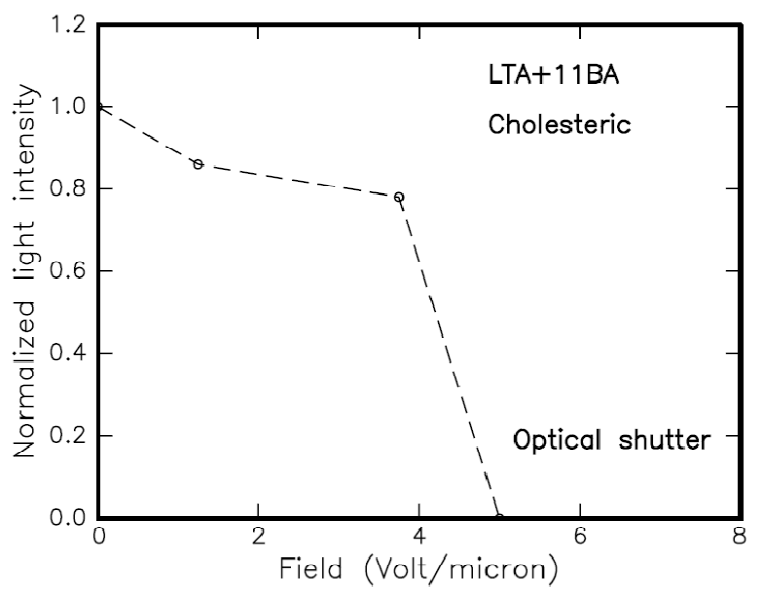

FIG. 9: Intensity profile of the cholesteric phase under various applied external bias voltages

\section{Intensity profile of the optical texture in FiT}

The sample LTA+11BA is filled in a commercially available buffed cell (Instec) and silver leads are drawn for con- 
tact. The intensity profile in various phases has been experimentally analyzed by applying external bias voltage of both polarities drawn from impedance analyzer (HP 4192A) and the intensity of the light from the liquid crystal sample is measured by a photo diode (TSL 252). As the external bias voltage is incremented in small steps, this in turn induced various FiT at different magnitudes of the applied voltage. The variation of the intensity of the texture is noted at each step of the applied bias voltage and plotted as shown in figure 9. It is not surprising to note that the magnitude of the light is almost unaltered in any of the induced transitions except in $\mathrm{E}_{0}$, and $E_{1}$ respectively. During the transition from $E_{0}$ and $E_{1}$ there is a steep sudden decrement of the intensity of light manifesting the distortion of the helix. In the this phase of FiT, which is referred as optical shutter, the optical profile is completely vanished. Thus the liquid crystal behaved as an optical shutter (plates 1-4). Hence this HBLC may be used as a light modulator.

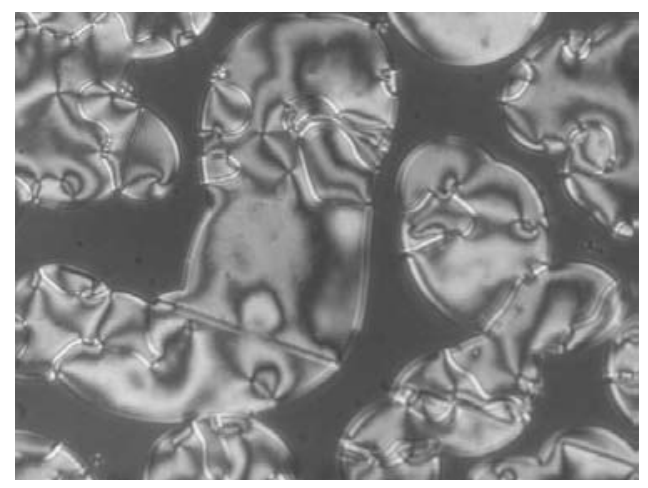

PLATE 1: Cholesteric phase with applied external bias voltage of 1.25 volts/micron

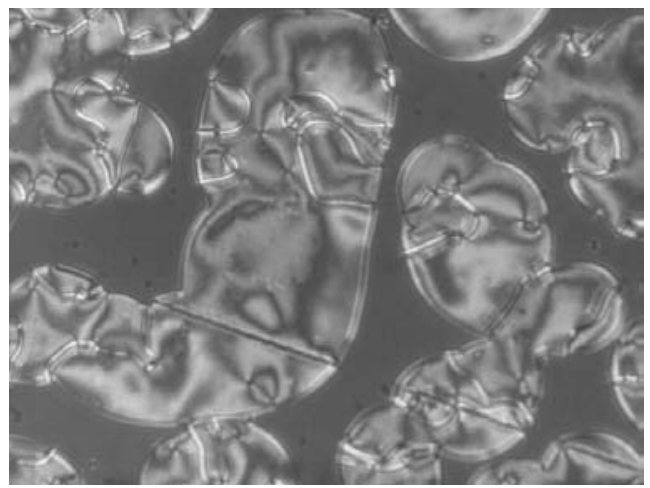

PLATE 2: Cholesteric phase with applied external bias voltage of 2.50 volts/micron

\section{Molecular modeling of field induced transitions}

A qualitative approach for the observed optical shutter phenomenon is described below. In the literature it is reported [44-48] the distortion and unwinding of the helicoidal struc-

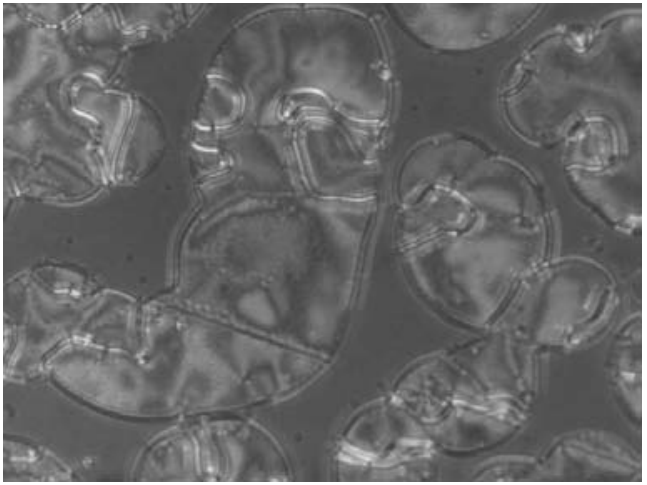

PLATE 3: Cholesteric phase with applied external bias voltage of 3.75 volts/micron

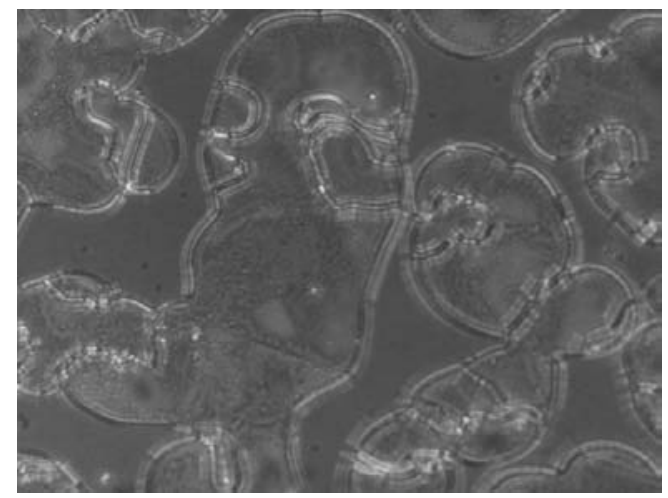

PLATE 4: Cholesteric phase with applied external bias voltage of 5.00 volts/micron

ture with applied field in the liquid crystal mesogen. The untwisting of the helical structure of a hydrogen bonded liquid crystal (HBLC) in a thin plane layer exposed to an external action (temperature or field) and its dependence on the molecular adhesive forces at the layer boundaries are responsible for field induced transitions. If the helical pitch is not infinite a non zero average polarization exists locally. When subjected to a weak electric fields the helix distorts [41] giving rise to small change in transmitted light as observed when the cell is placed under crossed polarizer. At much stronger fields there is a sudden transition such that the azimuthal orientation of the director is spatially uniform and all layers polarization is parallel to the applied field.

For very long pitch the liquid crystal behavior at small electric fields is expected to be different. The tilt plane of the molecules lies parallel to plane of the cell under these circumstances a continuous variation of the director orientation with field $(\mathrm{E}=0)$ begins. In this region the director profile is undisturbed. At the next level i.e. $\mathrm{E}=1$, the electric filed is sufficient to over come the elasticity possessed by the molecules. Molecules in adjacent layer undergo unequal azimuthal rotations resulting in a locally averaged nonzero polarization that couples to the electric filed. At much higher fields $(E=1)$ the distortion is more and it helps in rapid switching which is analogous to short helical pitch materials. The role of the anchoring energy is considerable at low fields, as the field increases the molecules get loosely bounded by the substrate 
surface. In other words the liquid crystal behaves as a light modulator. At high fields the extinction of the light is observed and this state may be referred as optical shutter. The parameters like helical pitch, anchoring energy and light modulation vary with applied field and govern the utility of the liquid crystal in display applications.

It has been experimentally observed from the helix studies that the magnitude of helical pitch decreases as one proceeds from the $E_{0}$ to $E_{1}$ and finally vanishes in $E_{1}$ phase. Thus it can be concluded that pitch in various field induced phase's orients depending on the strength of the applied bias. It is no surprise that the molecules prefer an alignment which resembles like homeotropic alignment, when the bias strength is increased to a maximum value and thus the molecules inhibits the light and act as a total optical shutter. The possible reasons for this field induced transitions can be elicited from the various surface anchoring energy [49] possessed by the liquid crystalline molecules.

\section{Acknowledgements}

One of the authors (MLNMM) acknowledges the financial support rendered by All India Council for Technical Education, Department of Science and Technology, and Defence Research Development Organization, New Delhi. Infrastructural support provided by Bannari Amman Institute of Technology is gratefully acknowledged.
[1] R.B. Meyer, L. Liebert, L. Strezelecki and P. Keller, J. Physique. Lett. 30, 69 (1975).

[2] T. Kato, (1998) Handbook of liquid crystals. Wiley-VCH: Weinheim.

[3] G.W. Gray , (1962) Molecular structure and properties of liquid crystals, Academic press, London.

[4] H. Kelker and R. Hatz, (1980) Handbook of liquid crystals. Verlag Chemie, Weinheim.

[5] T. Kato and J.M.J. Frechet, J. Am .Chem. Soc. 111, 8533 (1989).

[6] L. Yu, Liq. Cryst. 14, 1303 (1993).

[7] T. Kato, H. Kihara, T. Uryu, S. Ujiie, K. Iimura, J.M.J. Fréchet, U. Kumar, Ferroelectrics. 148, 161 (1993).

[8] T. Kato, T. Uryu, F. Kaneuchi, C. Jin and J.M.J. Fréchet, Liq. Cryst. 14, 1311 (1993).

[9] D. Demus, H. Demus and H. Zaschke, Flussige Kristalle (1974) Tabellen, VEB Deutcher Verlag fur Grundstoffindustrie; Leipzig.

[10] C.M. Paleos, D. Tsiourvas, Liq. Cryst. 28, 1127 (2001).

[11] B. Xu,. T .M .Swager, J. Am. Chem. Soc. 117, 5011 (1995).

[12] S. Malik, P.K. Dhal, R.A Mashelkar. 28, 2159 (1995).

[13] Z. Sideratou, D.Tsiourvas, C.M. Paleos and A. Skoulios, Liq. Cryst. 22,51 (1997)

[14] J.W. Goodby, R. Blinc, N.A Clark, S.T. Lagerwall, S.A. Osipov, S.A Pikin, T. Sakurai, Y. Yoshino and B. Zecks, (1991) Ferro Electric Liquid Crystal, Principles, Properties, and Applications. Philadalphia, U.S.A.Gorden and Breech Press.

[15] N.A. Clark and S.T. Lagerwall, Appl. Phys. Lett. 36, 899 (1980).

[16] H.R. Brand, P.E. Cladis and H. Pleiner, Macromol. 25, 7223 (1992).

[17] M.P. Petrov and L.V. Tsonev, Liq. Cryst. 21, 543 (1996).

[18] P.A. Kumar, M. Srinivasulu and V.G.K.M. Pisipati, Liq. Cryst. 26, 859 (1999).

[19] P. Swathi, P.A. Kumar and V.G.K.M. Pisipati , Liq. Cryst. 27, 665 (2000).

[20] M. Srinivasulu, P.V.V. Satyanarayana, P.A. Kumar and V.G.K.M. Pisipati, Liq. Cryst. 28, 1321 (2001).

[21] P. Swathi, S. Sreehari Sastry, P.A. Kumar, V.G.K.M. Pisipati Mol. Cryst. Liq. Cryst. 365, 523 (2001).

[22] B. Sreedevi, P.V. Chalapathi, M. Srinivasulu, V.G.K.M. Pisipati and D.M. Potukuchi, Liq. Cryst. 31, 303 (2004).

[23] T. Chitravel, M.L.N. Madhu Mohan, V. Krishnakumar, Mol Cryst. Liq.Cryst. 493, 17 (2008).

[24] V.N. Vijayakumar, K. Murugadass and M.L.N. Madhu Mohan, Mol. Cryst. Liq.Cryst, (2009). (Accepted for Publication).

[25] M.L.N. Madhu Mohan, B. Arunachalam, C. Arravindh Sankar
Metal and Mater. Trans. A, 39, 1192 (2008). M.L.N Madhu Mohan, B. Arunachalam Z.Naturforch, 63a, 435 (2008).

[26] Madhu Mohan M.L.N and V.G.K.M Pisipati, Liq. Cryst., 26, 1609 (2000)

[27] P.A. Kumar., M.L.N. Madhu Mohan and. V.G.K.M. Pisipati, Liq. Crys, 27, 1533 (2000).

[28] M.L.N. Madhu Mohan, P.A. Kumar, B.V.S.Goud and V.G.K.M. Pisipati, Mater. Res. Bull. 34, 2167 (1999).

[29] M.L.N. Madhu Mohan, P.A. Kumar and V.G.K.M. Pisipati, Ferroelectrics. 227, 105 (1999).

[30] V.N. Vijayakumar. and M.L.N. Madhu Mohan, Ferroelectrics (2009).accepted for publication.

[31] G.W. Gray and J.W.G. Goodby, Smetic Liquid Crystals - Textures and Structures. London, Leonard Hill (1984).

[32] C. Noot, S.P. Perkins, and H.J Coles, Ferroelectrics. 244, 331 (2000).

[33] P.G. de Gennes, (1974) The Physics of Liquid Crystals. Oxford Press, London.

[34] H.E. Stanley, (1971) Introduction to phase transition and critical phenomena. Clarendon Press.

[35] S. Kobayashi , S. Ishibashi, Mol. Cryst. Liq. Cryst., 257, 181 (1994).

[36] W. Jong-Guang, C. Shu-Hsia , Jap. J. Appl. Phys., 33, 6249 (1994).

[37] T .Qian, P.L. Taylor, Phys. Rev .E. 60, 2978 (1999).

[38] G. Napoli , J. Appl. Maths., 71, 34 (2006).

[39] L.J.M. Schlangen, P. Alexandre , and H..J. Cornelissen., J. Appl. Phys. 87, 3723 (2000).

[40] A.J. Hurd, S. Fraden, F. Lonberg, R.B. Meyer, J. de Physique, 46, 905 (1985).

[41] S.Zhang, B.Wen, S.S. Keast, M.E. Neubert, P.L. Taylor and C. Rosenblatt, Phys. Rev. Lett., 84, 4140 (2000).

[42] T.A. Rotinyan, E.I. Ryumtsev, S.B. Yazikov, JETP Lett., 46 , 417 (1987).

[43] P.E. Cladiss, T. Garel and P. Pieranski, Phys. Rev. Lett., 57, 2841, (1986).

[44] I. Abdulhalim , G. Moddel, Mol. Cryst. Liq. Cryst. 200, 79 (1991).

[45] L.A. Judge, E.E. Kriezis, S.J. Elston, Mol. Cryst. Liq. Cryst., 366, 661 (2001).

[46] P. Schiller and F. Zeitler, Phys. Rev. E., 56, 531 (1997).

[47] P. Schiller and F. Zeitler, J. de Physique II, 5, 1835 (1995).

[48] M. Petit, A.Daoudi M. Ismaili, and J.M. Buisine, Euro. Phys. J. E. 20, 327 (2006).

[49] J.O. Indekeu and A.N. Berker, Phys. Rev. A. 33, 1158 (1986). 